\title{
Safety, Efficacy and Cost Analysis of Rivaroxaban versus Fondaparinux for Thromboprophylaxis after Joint Replacement at an Inpatient Rehabilitation Facility
}

\author{
Nitika Agarwal ${ }^{1}$, Noel Rao ${ }^{1,2}$, Mary Mekheil ${ }^{1}$, Nadia Tancredi ${ }^{1}$ and Jeffrey Oken ${ }^{1,2}$ \\ 1. Marianjoy Rehabilitation Hospital, Wheaton, IL 60187, USA \\ 2. Department of PM\&R, Rosalind Franklin University of Medicine and Science, North Chicago, IL 60064, USA
}

\begin{abstract}
This study compares safety, efficacy, and cost of the new oral Factor Xa inhibitor rivaroxaban to fondaparinux, an injectable anticoagulant, for prevention of VTE (venous thromboembolism) after hip or knee arthroplasty within an IRF (inpatient rehabilitation facility). In this retrospective review, data was collected on the patients that were either status post total knee arthroplasty or total hip arthroplasty, admitted to the IRF over a 24 month period. All patients identified for inclusion in the study received either fondaparinux subcutaneously or rivaroxaban orally once daily as for VTE prevention. Primary efficacy outcomes were composite of any deep venous thrombosis, non-fatal PE (pulmonary embolism); and all-cause mortality. Primary safety outcomes were any major or non-major bleeding events. Analysis of 314 patient records (199 patients on rivaroxaban and 115 patients on fondaparinux) indicated no PE events. No DVT occurred in the patients prescribed rivaroxaban compared to $0.9 \%$ in the fondaparinux group. Major bleeding events occurred in $0.5 \%$ of patients prescribed rivaroxaban compared to $1.74 \%$ in fondaparinux group. Minor bleeding events occurred in $1 \%$ of patients prescribed rivaroxaban compared to $1.74 \%$ of patients in fondaparinux group. Direct acquisition cost analysis revealed $52 \%$ lower costs than fondaparinux, in the patients treated with rivaroxaban. In this study, rivaroxaban provided a safe and effective alternative to fondaparinux for prevention of VTE in post-operative total hip or knee replacement patients in the IRF setting. Rivaroxaban was also found to be favorable with respect to cost of acquisition, and ease of drug administration.
\end{abstract}

Key words: Rivaroxaban, fondaparinux, thromboprophylaxis, rehabilitation, arthroscopy, venous thromboembolism, cost analysis.

\section{Introduction}

VTE (Venous thromboembolism) comprising of DVT (deep venous thrombosis) and PE (pulmonary embolism) is a major and potentially fatal complication after major orthopedic surgery including THR (total hip replacement) and TKR (total knee replacement) [1]. DVT rates based on venography in clinical trials published from 1980 to 2002 in patient's receiving no thromboprophylaxis ranged from total percentage of 42 to 57 and proximal 18 to 36 for hip arthroplasty. For knee arthroplasty range was 41 to $85 \%$ total and proximal 5 to $22 \%$. PE rates derived from prospective studies that may have used thromboprophylaxis for hip

Corresponding author: Nitika Agarwal, Pharm.D., MBA, director of pharmacy, marianjoy rehabilitation hospital, E-mail: ndagarwal@yahoo.com. arthroplasty range from 0.9 to $2.8 \%$ with fatal PE 0.1 to $2 \%$. Whereas, after total knee arthroplasty rate reported ranged from 1.5 to $10 \%$ with fatal PE 0.1 to $1.7 \%$ [2]. Pharmacological thromboprophylaxis in patients undergoing THR and TKR significantly reduces the risk of postoperative VTE. In a study spanning over 13 year period, patients following major orthopedic surgery underwent VTE prophylaxis with LMWH (low-molecular-weight heparin) during their hospitalization only. VTE was diagnosed after discharge from hospital in $70 \%$ of patients who had developed this complication [3]. A systematic review of studies comparing prolonged duration (21 days or greater) with standard duration (7 to 10 days) thromboprophylaxis after major orthopedic surgery revealed prolonged prophylaxis decreased VTE without increasing major bleed but with increased risk 
for minor bleed [4].

A prospective multicenter study of 1,080 patients following THR and TKR pharmacological thromboprophylaxis was administered for a mean time of 36 days with incidences of $1.3 \%$ and $2.8 \%$ in hip and knee surgery patients. The rate of clinically significant bleeding was $1 \%$ [5]. In a multicenter study comparing effectiveness of adjusted dose warfarin and enoxaparin, VTE thromboprophylaxis provided only during hospitalization after total hip arthroplasty $0.3 \%$ managed with enoxaparin and $1.1 \%$ with warfarin had VTE during hospitalization. The benefit was lost after medication was discontinued with VTE $3.4 \%$ in enoxaparin and $2.6 \%$ in warfarin, with no difference in the prevalence of VTE between these 2 groups [6]. VTE prophylaxis following knee arthroplasty, $30 \mathrm{mg}$ of enoxaparin subcutaneously administered twice daily has been found to be superior to adjusted dose warfarin in reducing occurrence of asymptomatic DVT including proximal and distal. There was no significant difference between groups in the prevalence of major hemorrhage [7].

A meta-analysis of 4 randomized double-blind studies demonstrated efficacy of fondaparinux, a synthetic antithrombotic agent that inactivates factor $\mathrm{Xa}$ inhibiting thrombin generation over enoxaparin. Deep vein thrombosis prophylaxis was provided in patients with elective hip replacement, major knee surgery and surgery for hip fracture. The incidence of VTE by day 11 was $6.8 \%$ in Fondaparinux group and $13.7 \%$ in group treated with enoxaparin with overall risk reduction of VTE greater than 50\% without increasing the risk of clinically relevant bleeding [8].

In another study comparing efficacy and safety of fondaparinux with enoxaparin for prevention of VTE after elective major knee surgery, fondaparinux $2.5 \mathrm{mg}$ once daily was significantly more effective than enoxaparin $30 \mathrm{mg}$ twice daily. Risk reduction was $55.2 \%$ with no significant difference between the 2 groups in fatal bleeding into critical organs or bleeding requiring additional surgery, though major bleeding was more prevalent in those treated with fondaparinux [9]. In patients following surgery for hip fracture, VTE prophylaxis with fondaparinux had VTE incidence of $8.3 \%$ and enoxaparin $19.1 \%$ with reduction in risk with fondaparinux $56.4 \%$. These 2 groups had no significant differences in incidence of death or clinically relevant bleeding [10]. Enoxaparin and fondaparinux require parental administration and warfarin close monitoring due to unpredictable pharmacodynamics properties. These have been mainstay for thromboprophylaxis after major orthopedic surgery. Efficacy of the parentally administered indirect factor Xa inhibitor fondaparinux, interestingly enough, encouraged the development of direct factor Xa inhibitors [11].

In a randomized double-blind study efficacy and safety of rivaroxaban and enoxaparin for thromboprophylaxis after hip arthroplasty major VTE occurred in $0.2 \%$ of the rivaroxaban group and $2 \%$ in the enoxaparin group with absolute risk reduction of $1.7 \%$. Major bleeding occurred in $0.3 \%$ of the rivaroxaban group and $0.1 \%$ of the enoxaparin group. A once daily $10 \mathrm{mg}$ oral dose of rivaroxaban was significantly more effective for extended thromboprophylaxis than a once daily $40 \mathrm{mg}$ subcutaneous dose of enoxaparin with similar safety prophylaxis [12]. Another randomized double-blind trial compared rivaroxaban with enoxaparin after total knee arthroplasty. Major VTE occurred in $1 \%$ of rivaroxaban group and $2.6 \%$ of enoxaparin group with absolute risk reduction of $1.6 \%$. Major bleeding occurred in $0.6 \%$ of rivaroxaban group of patients and $0.5 \%$ of enoxaparin group. Rivaroxaban was superior to enoxaparin for thromboprophylaxis after total knee arthroplasty with similar rates of bleeding [13].

The studies enumerated above have compared dose adjusted warfarin to enoxaparin noting superiority of enoxaparin, enoxaparin to fondaparinux reporting superiority of fondaparinux, enoxaparin to rivaroxaban with greater effectiveness with similar safety profile of rivaroxaban over enoxaparin in VTE prophylaxis after hip and knee joint replacement surgery. There are no 
studies reported in the literature comparing safety, efficacy and costs of rivaroxaban versus fondaparinux. The purpose of this study is to compare safety, efficacy and cost of the new oral factor Xa inhibitor rivaroxaban to fondaparinux, for prevention of VTE (venous thromboembolism) after hip or knee arthroplasty, within an IRF (inpatient rehabilitation facility).

\section{Methods}

\subsection{Patient Population}

This retrospective cohort study was performed at a 128 bed free-standing academic IRF (inpatient rehabilitation facility). Data was collected on the patients that were either status post total knee arthroplasty or total hip arthroplasty, admitted to the IRF over a 24 month period ranging from January 2011 to December 2012. All patients identified for inclusion in the study received either fondaparinux $2.5 \mathrm{mg}$ subcutaneously or rivaroxaban $10 \mathrm{mg}$ orally once daily as the primary anticoagulant agent for VTE prevention. Fondaparinux was the primary agent on formulary until October 2011, and rivaroxaban became primary formulary agent since November 2011. Patients receiving any other anticoagulant or long term anticoagulation at admission or being treated for DVT and or PE were excluded. A comprehensive chart review was conducted for all patients included in the study. Patients were grouped according to the primary anticoagulant received and that met the inclusion criteria. This study was conducted in compliance with institutional review board requirements at Marianjoy Rehabilitation Hospital.

\subsection{Data Collection}

Demographic data, admission diagnosis, medication and the number of doses received, length of hospitalization, chart documentation of bleeding, and DVT/PE were collected by the investigators using a standard data collection sheet. Data was collected by performing comprehensive chart reviews. Drug cost data collections were based on the WAC (wholesale acquisition costs) for rivaroxaban and generic fondaparinux (average WAC for generic fondaparinux as of December 2012). Cost comparison was done by calculating cost of total doses of rivaroxaban dispensed and the cost of equal number of fondaparinux doses. Event related costs due to DVT/PE or bleeding were not analyzed.

\subsection{Outcome Measures}

Primary effectiveness outcomes were composite of any DVT (deep venous thrombosis), proximal and/or distal; confirmed by venous Doppler study in clinically symptomatic patients; non-fatal, symptomatic, objectively confirmed PE (pulmonary embolism); and all-cause mortality. Primary safety outcomes were any major or non-major bleeding events. Major bleeding event was defined as fatal bleeding, symptomatic bleeding into critical organ, bleeding into surgical site/joint, clinically overt bleeding leading to fall in hemoglobin of greater than or equal to $2 \mathrm{~g} / \mathrm{dL}$ or bleeding leading to transfusion of 2 or more units of packed red blood cells. Minor bleeding events were defined as epistaxis, gingival bleed, hematochezia, hematuria, or clinically symptomatic anemia requiring anticoagulant to be discontinued.

\section{Results}

\subsection{Study Populations}

A total of 314 patient records were analyzed during the study period between January 2011 and December 2012. Of these, 199 (63\%) patients received rivaroxaban and, 115 (37\%) received fondaparinux. Baseline characteristics were similar in both groups, and the data on demographics, admission diagnosis, and length of hospitalization/stay is presented in Table 1.

\subsection{Efficacy and Safety Outcomes}

No PE events occurred in either group during their inpatient rehabilitation stay. No DVT occurred in the patients in the rivaroxaban group and $1 \mathrm{DVT}$ occurred 
Table 1 Baseline characteristics of the 314 patients in the study.

\begin{tabular}{|c|c|c|c|}
\hline Characteristic & Rivaroxaban & Fondaparinux & Overall \\
\hline Total number of patients & 199 & 115 & 314 \\
\hline Males & 70 & 28 & 98 \\
\hline Females & 129 & 87 & 216 \\
\hline Average Age (yrs) & 69.6 & 71.1 & 69.5 \\
\hline Average $\operatorname{LOS}^{1}$ (days) & 10.3 & 10.6 & 10.5 \\
\hline TKA & 131 & 45 & 176 \\
\hline THA & 68 & 70 & 138 \\
\hline
\end{tabular}

${ }^{1}$ length of stay

\section{Efficacy and Safety Outcomes Comparison}

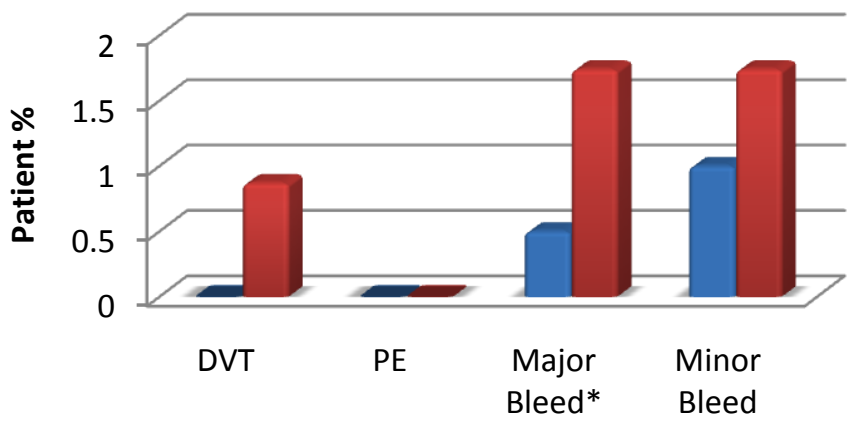

Rivaroxab

an

Fondapari nux

Primary Outcomes

Fig. 1 Efficacy and safety outcomes.

*Major bleed in the rivaroxaban group was unclear.

out of 115 patients $(0.9 \%)$ in the fondaparinux group.

Major bleeding events occurred in 1 of 199 patients $(0.5 \%)$ who received rivaroxaban and 2 of $115(1.74 \%)$ who received fondaparinux. No events of fatal bleeding occurred. Of the major bleeding event identified in rivaroxaban group, rivaroxaban was discontinued post coffee ground emesis and changed to enoxaparin. Two days later there was a drop in hemoglobin and the patient received 2 units of blood transfusion. Subsequently patient was restarted on rivaroxaban without further adverse event. It was unclear if the bleed was due to rivaroxaban. Of the two major bleeding events identified with patients prescribed fondaparinux, both required blood transfusions and the drug was discontinued in one patient but continued in the other.
Minor bleeding events occurred in 2 of 199 patients (1\%) who received rivaroxaban and 2 of 115 patients (1.74\%) who received fondaparinux. Of the minor bleedings events identified with patients prescribed rivaroxaban, anti-coagulant was discontinued due to hemoglobin being low in one case and continued in the other. Of the minor bleeding events identified with patients prescribed fondaparinux, anti-coagulant was discontinued in one due to symptomatic anemia and continued in the other.

\subsection{Cost Outcomes}

The duration for thromboprophylaxis for both anticoagulants was 10 days on average and rivaroxaban was given as $10 \mathrm{mg}$ once daily while fondaparinux was given as $2.5 \mathrm{mg}$ daily. The Drug cost for rivaroxaban 
$10 \mathrm{mg}$ at $\$ 6.39$ per day were $\$ 11,994$ over the course of study period (1,877 doses dispensed for 199 patients). Daily drug cost for fondaparinux $2.5 \mathrm{mg}$ were $\$ 13.20$ per patient, estimated to be $\$ 24,767$ for 1877 doses (that were dispensed as rivaroxaban). Cost savings were calculated as approximately $\$ 13,000$ $(\$ 24,767-\$ 11,994)$ for 199 patients for the period of thromboprophylaxis. Switching the 1000-patient cohort from generic fondaparinux to rivaroxaban would result in potential cost savings of $\$ 68,100$ over the course of thromboprophylaxis.

\section{Discussion}

While previous studies have reported greater effectiveness with similar safety profile of rivaroxaban over enoxaparin, this study is the first one to compare safety, efficacy and cost comparison between fondaparinux and rivaroxaban.

After reviewing the details on patients that had major bleeds, it was found that rivaroxaban was restarted in one patient and fondaparinux was discontinued in one and restarted in another. The patient who was on rivaroxaban had an emergency transfer to acute care facility due to 3 episodes of dark emesis (coffee ground), severe constipation, ileus, and general abdominal pain. She did not have blood in the emesis and had never had GI bleed in past, no melena and hematochezia were noted. Upon admission to IRF patient's hemoglobin was $8.4 \mathrm{gm} / \mathrm{dL}$ which changed to $9.1 \mathrm{gm} / \mathrm{dL}$ upon discharge to acute care facility. Review of medications indicated no other agent that would increase the risk of bleeding, patient was not on any interacting medications or medications that would contribute to bleeding risk. Per records from acute care hospital where patient was transferred it was noted that since the patient had ileus the oral feedings were discontinued and patient was placed on intravenous fluids. Rivaroxaban was discontinued and it was switched to enoxaparin $30 \mathrm{mg}$ subQ twice daily for anticoagulation and the hemoglobin fell from 9.8 $\mathrm{gm} / \mathrm{dL}$ to $7.8 \mathrm{gm} / \mathrm{dL}$ in 2 days at which point patient received 2 units of blood. The overall assessment and impression indicated likely dilutional and blood loss anemia from the TKA and post-operative ileus. Patient returned to IRF 4 days later and was resumed on rivaroxaban without further complications. Patient continued rehabilitation for 10 days and the hemoglobin upon discharge was $10.5 \mathrm{gm} / \mathrm{dL}$. Thus it was unclear if the acute anemia was related to rivaroxaban, or enoxaparin, or due to surgery.

Of the 2 patients that experienced major bleed in the fondaparinux group one patient had drop in hemoglobin from $8.1 \mathrm{gm} / \mathrm{dL}$ upon admission to 7.0 $\mathrm{gm} / \mathrm{dL}$ three days later. Patient was sent out to acute care facility for 2 units of packed red blood cell transfusion. Per orders from orthopedic surgeon, fondaparinux was discontinued and patient was started on aspirin $325 \mathrm{mg}$ two times daily. Patient progressed and completed rehabilitation goals without any further complications. The second patient who experienced major bleed in the fondaparinux group had a drop in hemoglobin from $9.1 \mathrm{gm} / \mathrm{dL}$ prior to admission to 7.2 $\mathrm{gm} / \mathrm{dL}$ on admission to $6.8 \mathrm{gm} / \mathrm{dL}$ after admission. Patient was transfused with 2 units of packed red blood cells. Fondaparinux was held for 4 days and later restarted per attending physician's order, as the impression was the patient had acute blood loss due to surgery and not due to the drug.

\section{Conclusion}

In this study, rivaroxaban, an oral direct Factor $\mathrm{Xa}$ inhibitor, provided a safe and effective alternative to fondaparinux, an injectable Factor Xa inhibitor for prevention of venous thromboembolism in post-operative total hip or knee replacement patients in the inpatient rehabilitation hospital setting. Both rivaroxaban and fondaparinux were equally effective in preventing VTE; however fondaparinux acquisition cost was higher than rivaroxaban cost in addition to its parenteral cost of administration. Rivaroxaban was found to be favorable with respect to cost of acquisition, and ease of drug administration. 


\section{after Joint Replacement at an Inpatient Rehabilitation Facility}

\section{References}

[1] Falck-Ytter, Y., Francis, C. W., Johanson, N. A., Curley, C., Dahl, O. E., Schulman, S., Ortel, T. L., Pauker, S. G., and Colwell, C. W. Jr. 2012. "American College of Chest Physicians Prevention of VTE in Orthopedic Surgery Patients, Antithrombotic Therapy and Prevention of Thrombosis." 9th Edition: American College of Chest Physicians, Evidence-Based Clinical Practice Guidelines. Chest 141: e278S-e325S.

[2] Geerts, W. H., Pineo, G. F., Heit, J. A., Bergqvist, D., Lassen, M. R., Colwell, C. W., and Ray, J. G. 2004. "Prevention of Venous Thromboembolism: The 7th ACCP Conference on Antithrombotic and Thrombolytic Therapy." Chest 126: 338S-400S.

[3] Bjornara, B. T., Gudmundsen, T. E., and Dahl, O. E. 2006. "Frequency and Timing of Clinical Venous Thromboembolism after Major Joint Surgery." The Journal of Bone and Joint Surgery (BR) 88-B: 386-91.

[4] Sobieraj, D. M., Lee, S., Coleman, C. I., Tongbram, V., Chen, W., Colby, J., Kluger, J., Makanji, S., Ashay, A. O., and White, M. C. 2012. "Prolonged versus Standard Venous Thromboprophylaxis in Major Orthopedic Surgery, a Systematic Review." Annals of Internal Medicine 156 (10): 720-7.

[5] Samama, C. M., Ravaud, P., Parent, F., Barre, J., Mertl, P., and Mismetti, P. 2007 "Epidemiology of Venous Thromboembolism after Lower Limb Arthroplasty; FOTO Study." Journal of Thrombosis and Hemostasis 5: 2360-7.

[6] Colwell, C. W. Jr., Collis, D. K., Paulson, R., McCutchen, J. W., Bigler, G. T., Lutz, S., and Hardwick, M. E. 1990. "Comparison of Enoxaparin and Warfarin for the Prevention of Venous Thromboembolic Disease after Total Hip Arthroplasty." The Journal of Bone and Joint Surgery 81 A (7): 932-40.

[7] Fitzgerald, R. H., Spiro, T. E., Trowbridge, A. A., Gardner,
G. A. Jr, Whitsett, T. L., O'Connell, M. B., Ohar, J. A., and Young, T. R. 2001 "Prevention of Venous Thromboembolic Disease Following Primary Total Knee Arthroplasty." Journal of Bone and Joint Surgery 83A, (6): 900-6.

[8] Turpie, A. G. G., Bauer, K. A., Eriksson, B. I., and Lassen, M. R. 2002 "Fondaparinux versus Enoxaparin for the Prevention of Venous Thromboembolism in Major Orthopedic Surgery. A Meta-analysis of 4 Randomized Double-Blind Studies." Arch. Intern. Med. 162: 1833-40.

[9] Bauer, K. A., Eriksson, B. I., Lassen, M. R., and Turpie, A. G. G. 2001 "Fondaparinux Compared with Enoxaparin for the Prevention of Venous Thromboembolism after Elective Major Knee Surgery." New England Journal of Medicine 345 (18): 1305-10.

[10] Eriksson, B. I., Bauer, K. A., Lassen, M. R., and Turpie, A. G. G. 2001 "Fondaparinux Compared with Enoxaparin for the Prevention of Venous Thromboembolism after Hip Fracture Surgery." New England Journal of Medicine 345: 1298-304.

[11] Turpie, A. G. G. 2007 “Oral, Direct Factor Xa Inhibitors in Development for the Prevention and Treatment of Thromboembolic Diseases." Arterioscler Thromb Vasc. Biol. 27: 1238-47.

[12] Eriksson, B. I., Borris, L. C., Friedman, R. J., Haas, S., Huisman, M. V., Kakar, A. K., Bandel, T. J., Beckmann, H., Muehlhofer, E., Misselwitz, F., and Geerts, W. 2008 "Rivaroxaban versus Enoxaparin for the Thromboprophylaxis after Hip Arthroplasty." New England Journal of Medicine 358: 2765-75.

[13] Lassen, M. R., Ageno, W., Borris, L., Leiberman, J. R., Rosencher, N., Bandel, T. J., Misselwitz, F., and Turpie, A. G. G. 2008 "Rivaroxaban versus Enoxaparin for Thromboprophylaxis after Total Knee Arthroplasty." New England Journal of Medicine 358: 2776-86. 